\title{
Is vitamin D status associated with open-angle glaucoma? A cross-sectional study from South Korea
}

\author{
Tae Keun Yoo ${ }^{1,2, *}$, Ein $\mathrm{Oh}^{1}$ and Samin Hong ${ }^{3, *}$ \\ 'Department of Medicine, Yonsei University College of Medicine, 50 Yonsei-ro, Seodaemun-gu, Seoul 120-752, \\ Republic of Korea: ${ }^{2}$ Department of Medical Engineering, Yonsei University College of Medicine, Seoul, Republic \\ of Korea: ${ }^{3}$ Institute of Vision Research, Department of Ophthalmology, Yonsei University College of Medicine, \\ Seoul, Republic of Korea
}

Submitted 10 July 2013: Final revision received 13 October 2013: Accepted 18 November 2013: First published online 10 January 2014

\begin{abstract}
Objective: Open-angle glaucoma (OAG) is one of the major chronic diseases involving the optic nerve. However, little is known about the association between vitamin D and OAG. The present study was conducted to test the hypothesis that lower vitamin D status is associated with greater prevalence of OAG.

Design: Cross-sectional study. Multivariable logistic regression was performed to examine the relationship between serum 25-hydroxyvitamin D (25(OH)D) and OAG after adjusting for traditional potential confounders. OAG was defined by the criteria of the International Society for Geographical and Epidemiological Ophthalmology.

Setting: The Fifth Korean National Health and Nutrition Examination Survey conducted in 2010-2011.

Subjects: Six thousand and ninety-four adult participants randomly selected from 192 surveys in 131 locations in South Korea.

Results: Multivariable-adjusted odds ratios of OAG across quintiles of decreasing $25(\mathrm{OH}) \mathrm{D}$ were $1 \cdot 26,1 \cdot 00$ (reference), $1 \cdot 31,1.36$ and 1.69 ( $P$ for quadratic trend $<0 \cdot 01)$. The odds ratio for the lowest $25(\mathrm{OH}) \mathrm{D}$ quintile was significantly higher than that for the second quintile $(P<0 \cdot 01)$. In addition, we discovered that the predictors for worsening of OAG, such as intraocular pressure or vertical and horizontal cup-to-disc ratios, had a significant relationship with $25(\mathrm{OH}) \mathrm{D}$ level. Conclusions: There was a reverse J-shaped association between $25(\mathrm{OH}) \mathrm{D}$ levels and the risk of OAG, with significantly elevated risk at lower $25(\mathrm{OH}) \mathrm{D}$. The findings of this research suggest that vitamin D deficiency should be considered as a potential risk factor for the development of OAG. To our knowledge, the present study is the first one that shows an association between vitamin D status and $\mathrm{OAG}$.
\end{abstract}

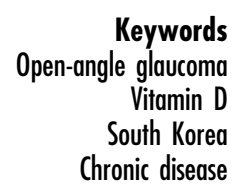

Open-angle glaucoma (OAG) is one of the major chronic diseases involving the optic nerve. It is the leading cause of irreversible blindness and a recent report suggested that by 2020 the disease will affect approximately 59 million people globally ${ }^{(1)}$. If OAG is not treated timely, severe vision loss can result from progressive optic neuropathy. Generally, OAG is characterized by progressive visual field loss and glaucomatous optic disc change with loss of retinal nerve fibre tissues ${ }^{(2)}$. Since these processes are chronic and irreversible, medical practitioners are striving to understand the risk factors associated with the development of glaucoma.

There are two types of glaucoma: angle-closure glaucoma (ACG) and OAG. While it is known that the damage to the optic nerve due to ACG results from obstruction of the outflow of aqueous humour, with the elevation of intraocular pressure (IOP), the pathophysiology of OAG is not yet fully understood ${ }^{(2)}$. Recent studies have described that OAG is not only an IOP-induced disease of the optic nerve, but also an age-related and neurodegenerative chronic disease ${ }^{(3,4)}$. IOP-induced stress, such as elevated IOP and fluctuation of IOP, compresses the structures around the optic nerve and can lead to damage in the retina and the optic nerve by disturbing the axonal transport of trophic molecules within the nerve fibres ${ }^{(2)}$. Emerging evidence is showing that poor ocular blood flow and neurodegenerative change also contribute to OAG pathophysiology ${ }^{(4)}$. Poor ocular blood flow due to vascular dysfunction may cause OAG by chronic ocular ischaemia and interruption of nutrient supply to the optic nerve ${ }^{(5)}$. 
Neurodegenerative effects due to ageing, inflammation and metabolic stress have also been reported as factors contributing to glaucomatous optic neuropathy ${ }^{(6)}$. All these risk factors influence each other and have complex effects on the development of OAG.

Recently, vitamin D has become a major interest area of medical researchers. Vitamin D is an important secosteroid hormone that plays a role in the signalling pathways related to bone and mineral metabolism, cellular proliferation, immune modulation and the oxidative-stress system $^{(7,8)}$. In general, 25-hydroxyvitamin D (25(OH)D) is accepted as the most reliable biomarker for assessing individual vitamin D status. Based on the results of serum 25(OH)D measurements in large population-based studies, it is seen that vitamin D deficiency may be associated with neurodegenerative effects on the central nervous system $^{(9,10)}$. Several biological experiments have suggested that vitamin D regulates the functions of neuroprotection in the central nervous system, including in the optic nerve ${ }^{(11,12)}$. Moreover, vitamin D status can also affect chronic metabolic diseases, including diabetes, hypertension and dyslipidaemia, which are considered as the important metabolic risk factors of elevated IOP and reduced ocular blood flow ${ }^{(4,13)}$. Therefore, it can be hypothesized that vitamin D status is associated with the development of chronic optic neuropathy, i.e. OAG. However, until now, little is known concerning the epidemiological association of vitamin D status with OAG.

The present study was conducted to test the hypothesis that lower vitamin D status is associated with a greater prevalence of OAG. We investigated the relationship between the presence of OAG and serum 25(OH)D level, with adjustment for traditional potential confounders, using information from a cross-sectional, nationwide health survey. In addition, to elucidate the effects on glaucomatous changes, we examined serum 25(OH)D level in relation to IOP, which is considered to be the most important predictor of glaucoma, and vertical and horizontal cup-to-disc ratios of the optic nerve head, which reflect the structural change of the optic nerve head ${ }^{(2,14)}$.

\section{Methods}

\section{Study population}

The current study was based on health records collected from the Fifth Korean National Health and Nutrition Examination Survey (KNHANES V; http://knhanes.cdc. go.kr/knhanes) conducted in 2010 and 2011. The KNHANES $\mathrm{V}$ is a nationwide and population-based crosssectional survey that was conducted by the Division of Chronic Disease Surveillance, Korea Centers for Disease Control and Prevention ${ }^{(15)}$. The KNHANES V consists of health records based on a health interview, a health examination and a nutritional survey. All participants for KNHANES V were randomly selected from 192 surveys in
131 locations using stratified sampling, considering population, gender, age, regional area and type of residential area. The protocol for our study was approved by the institutional review board at the Korea Centers for Disease Control and Prevention, Seoul, South Korea. All participants signed forms consenting to the use of their health information for the study.

A total of 17476 participants were enrolled in the KNHANES $\mathrm{V}$. We restricted the study population to middle-aged and elderly participants, who are generally considered at high risk of $\mathrm{OAG}^{(16)}$. We excluded participants younger than 45 years of age $(n 9607)$ and those not having an eye examination done ( $n$ 466). Participants with age-related macular degeneration and diabetic retinopathy ( $n$ 872) were excluded, since these diseases are the major causes of disturbance in visual field testing for the diagnosis of glaucoma ${ }^{(17)}$. To investigate a specific association between vitamin $\mathrm{D}$ and $\mathrm{OAG}$, we also excluded participants without a $25(\mathrm{OH}) \mathrm{D}$ measure $(n$ 432) and with ACG ( $n$ 5). Finally, 6094 participants were eligible for the current study (Fig. 1).

\section{Opbthalmologic examination}

A structured slit-lamp examination for evaluation of glaucoma status was performed. The slit-lamp examination was performed for determination of diseases in the anterior segment of the eye and measurement of the IOP and anterior chamber depth using the Van Herick method. A digital non-mydriatic fundus camera and a Nikon D-80 digital camera (Nikon Inc., Tokyo, Japan) were used to obtain the digital fundus images. Digital images were captured from all participants under physiological mydriasis. Visual field testing using Humphrey Matrix (Carl Zeiss Meditec Inc., Dublin, CA, USA) was performed if the participants had elevated IOP ( $\geq 22 \mathrm{mmHg}$ ) or a glaucomatous optic disc. The glaucomatous optic disc included any of the following four conditions: (i) vertical or horizontal cup-to-disc ratio $\geq 0 \cdot 5$; (ii) presence of optic disc haemorrhage; (iii) presence of retinal nerve fibre layer defect; and (iv) violation of the ISNT rule (that normal eyes show a characteristic configuration for disc rim thickness of inferior $>$ superior $>$ nasal $>$ temporal). All ophthalmologic examinations were done by a trained ophthalmologist or ophthalmology resident ${ }^{(18)}$.

Finally, the participants were classified into three groups: (i) healthy control, (ii) glaucoma suspect and (iii) OAG. Participants with OAG were defined as meeting the diagnostic criteria of the International Society for Geographical and Epidemiological Ophthalmology (ISGEO) ${ }^{(19,20)}$. The specific diagnostic criteria adopted for the Korean population were as follows ${ }^{(18)}$ : (i) the presence of frequency doubling technology (FDT) testing results, fixation error and false positive error $\leq 1$; (ii) glaucomatous optic disc (loss of neuro-retinal rim with vertical or horizontal cup-to-disc ratio $\geq 0 \cdot 6$, or presence of optic disc haemorrhage, or presence of retinal nerve fibre layer defect, or violation of the ISNT rule); 


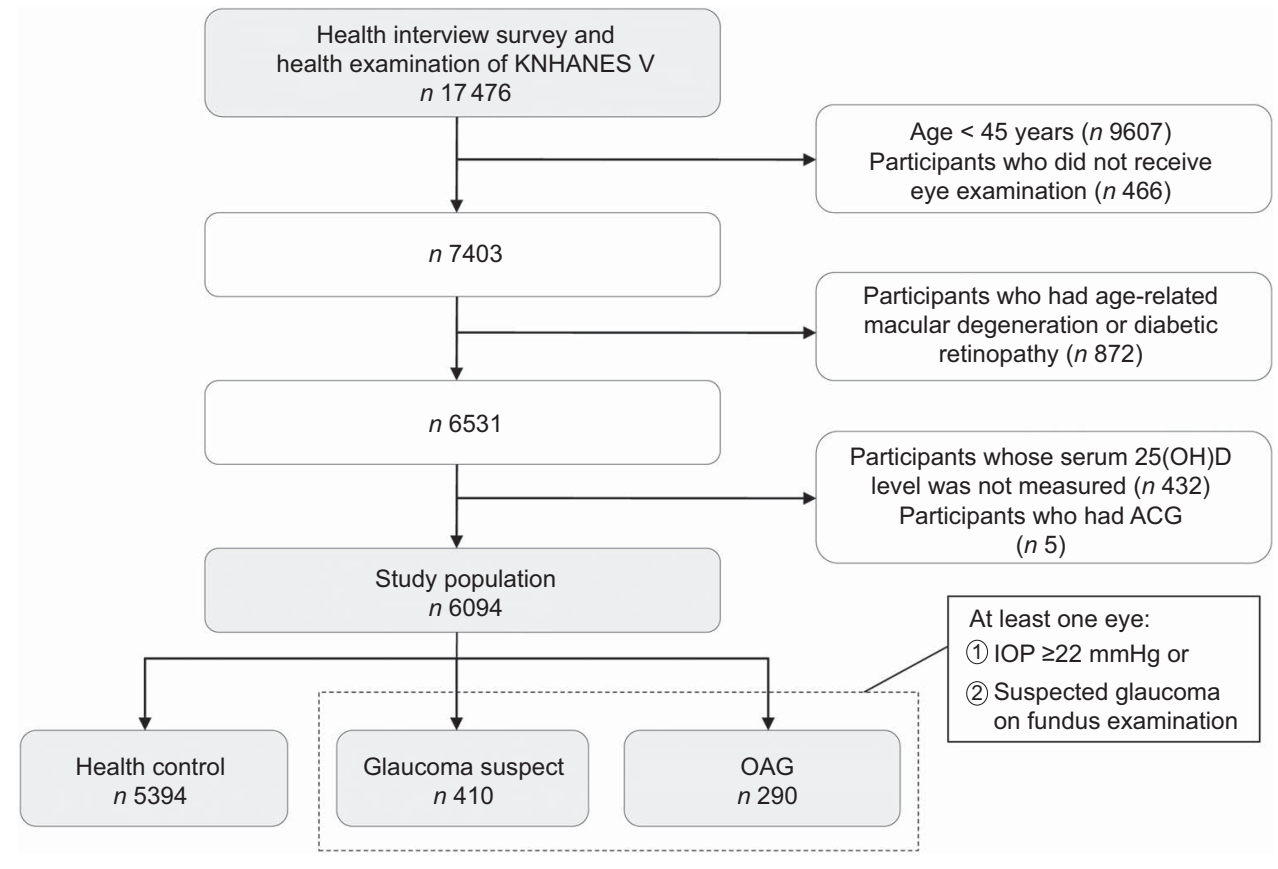

Fig. 1 Flow diagram of inclusion or exclusion of study participants from the Fifth Korean National Health and Nutrition Examination Survey (KNHANES V). OAG, open-angle glaucoma; 25(OH)D, 25-hydroxyvitamin D; ACP, angle-closure glaucoma; IOP, intraocular pressure

(iii) presence of an abnormal FDT testing result (at least one location of reduced sensitivity); and (iv) the presence of an open angle (peripheral anterior chamber depth $>1 / 4$ corneal thickness). Glaucoma suspects, who had structural glaucomatous change without visual field defect, were defined as having elevated IOP ( $\geq 22 \mathrm{mmHg}$ ) or a glaucomatous optic disc without OAG. When both eyes were eligible, one eye was randomly selected and used for the analyses.

\section{Measurement of serum 25-bydroxyvitamin $D$}

Fasting blood samples of individual participants were collected for measurements of serum $25(\mathrm{OH}) \mathrm{D}$ concentration. Blood samples were immediately refrigerated and transported to the Central Testing Institute in Seoul, South Korea. Blood samples were processed and analysed within $24 \mathrm{~h}$ after transportation. Serum 25(OH)D levels were measured using a $\gamma$ counter (1470 Wizard; Perkin-Elmer Finland) with a RIA (Dia-Sorin, Stillwater, MN, USA). The inter-assay CV were $11 \cdot 7 \%, 10 \cdot 5 \%, 8 \cdot 6 \%$ and $12 \cdot 5 \%$ at $8 \cdot 6,22 \cdot 7,33 \cdot 0$ and $49 \cdot 0 \mathrm{ng} / \mathrm{ml}$, respectively ${ }^{(21)}$. For the present study, 25(OH)D was categorized into quintiles: $\geq 23.79 \mathrm{ng} / \mathrm{dl}$ (highest group, median: $27 \cdot 41 \mathrm{ng} / \mathrm{dl}$ ); $19 \cdot 50-23 \cdot 78 \mathrm{ng} / \mathrm{dl}$ (median: $21 \cdot 29 \mathrm{ng} / \mathrm{dl}$ ); $16 \cdot 33-19 \cdot 49 \mathrm{ng} / \mathrm{dl}$ (median: $17 \cdot 77 \mathrm{ng} / \mathrm{dl}$ ); 13.09-16.32 ng/dl (median: $14 \cdot 77 \mathrm{ng} / \mathrm{dl}$ ); and $\leq 13 \cdot 08 \mathrm{ng} / \mathrm{dl}$ (lowest group, median: $11 \cdot 00 \mathrm{ng} / \mathrm{dl}$ ).

\section{Assessment of covariates}

Information on traditional glaucoma-related risk factors was obtained from face-to-face interviews. Each participant completed a questionnaire by providing information on age, smoking status (current smoker and non-smoker), sun exposure $(\geq 2 \mathrm{~h} / \mathrm{d}$ and $<2 \mathrm{~h} / \mathrm{d}$ ), family history of glaucoma, migraine and cold hands. These confounding factors were identified from a previous study of a Korean population-based sample ${ }^{(22)}$. Visual acuity was measured at a distance of $4 \mathrm{~m}$ using an international standard vision chart based on the LogMAR scale, which is an arithmetic progression with better acuity scoring a low number and worse acuity scoring a high number ${ }^{(23)}$. Since impaired visual acuity is a risk factor for reduced physical function and can affect lifestyle associated with vitamin D synthesis and intake, visual acuity was recognized as a confounding factor $^{(24)}$. It is widely recognized that metabolic status also contributes to the incidence of $\mathrm{OAG}^{(4)}$. Therefore, we included blood pressure and several laboratory findings as confounders. Fasting blood samples were analysed in local community health centres. Measurements of serum biomarkers, including fasting glucose, total cholesterol and TAG, were taken. Blood pressure was also measured by the health professionals. To avoid collinearity between systolic and diastolic blood pressure in multivariable regression models, we selected systolic blood pressure as a covariate. When calculating the correlation matrix of all confounding variables, there was no multi-collinearity problem (all pair-wise Pearson's correlations $r<0.5$ ).

\section{Statistical analysis}

Characteristics of the participants according to the category of glaucoma status were compared using the $\chi^{2}$ test for categorized data and one-way ANOVA for continuous data. In multivariable analyses, we established three 
adjusted models. Model 1 was adjusted for sex, age, current smoking status, sun exposure, family history of glaucoma, migraine and cold hands. Model 2 was adjusted for variables in Model 1 plus metabolic factors which included systolic blood pressure, fasting glucose, total cholesterol and TAG. In Model 3, further adjustments were made for visual acuity and IOP. To assess the association of serum 25(OH)D level with OAG, we first used multivariable logistic regression to estimate the relevant odds ratios by $25(\mathrm{OH}) \mathrm{D}$ quintile; in this analysis we studied the two clinical outcomes: (i) OAG and (ii) glaucoma suspect or OAG. When a non-linear relationship was observed, a quadratic trend analysis was employed. In addition, we fitted generalized additive models with cubic spline regression to visualize the dose-response association between serum 25(OH)D level and OAG. The resulting spline models represented log odds ratio of OAG adjusted for variables in Model 3. We performed ANCOVA to calculate geometric means of IOP, vertical and horizontal cup-to-disc ratios according to $25(\mathrm{OH}) \mathrm{D}$ quintile, after adjusting for potential confounders. Geometric means were computed by performing the regression of the natural log-transformed values to improve approximation of a skewed distribution and taking back-transformation of the resulting log-transformed values. Multivariable linear regression models also were used to assess the association of continuous serum $25(\mathrm{OH}) \mathrm{D}$ level with IOP, vertical and horizontal cup-to-disc ratios. To test for the linear and quadratic trends across increasing categories of $25(\mathrm{OH}) \mathrm{D}$, we treated the categories of each quintile as a simple continuous variable in a multivariable-adjusted model. We squared a centred linear trend variable of each quintile to assess quadratic trend ${ }^{(25)}$. The Wald $\chi^{2}$ test was used to test for interaction. We considered $P<0.05$ as the indicator of statistical significance. All statistical analyses were completed using the statistical software packages IBM SPSS Statistics 18 and R software version 3.0.2 (The Comprehensive R Archive Network; http:// cran.r-project.org).

\section{Results}

Characteristics of the participants by the category of glaucoma status are shown in Table 1 . Our study population consisted of $5394(88.5 \%)$ healthy controls, $410(6.7 \%)$ with glaucoma suspect and 290 (4.8\%) with OAG. While some confounding variables, namely sex, age, family history of glaucoma, systolic blood pressure and visual acuity, differed significantly among the groups categorized by glaucoma status, there was no significant difference in serum 25(OH)D level. However, when the categorization was done by the quartiles of age, we observed a reverse J-shaped pattern with a slightly elevated proportion of participants with OAG in the first $25(\mathrm{OH}) \mathrm{D}$ quintile $(\geq 23.79 \mathrm{ng} / \mathrm{dl})$, the lowest proportion in the second quintile $(19 \cdot 50-23 \cdot 78 \mathrm{ng} / \mathrm{dl})$, and gradual increases for the third (16.33-19.49ng/dl), fourth (13.09-16.32 ng/dl) and fifth quintiles ( $\leq 13 \cdot 08 \mathrm{ng} / \mathrm{dl}$; Fig. 2).

From the observation above, we hypothesized that there would be a quadratic relationship between serum 25(OH)D level and OAG. Table 2 shows the odds ratios of

Table 1 Characteristics of individuals with healthy control, glaucoma suspect and open-angle glaucoma eyes among 6094 adult participants in the Fifth Korean National Health and Nutrition Examination Survey (KNHANES V), 2010-2011

\begin{tabular}{|c|c|c|c|c|c|c|c|}
\hline & \multicolumn{2}{|c|}{$\begin{array}{l}\text { Healthy control } \\
\quad(n \text { 5394) }\end{array}$} & \multicolumn{2}{|c|}{$\begin{array}{l}\text { Glaucoma suspect } \\
\qquad(n 410)\end{array}$} & \multicolumn{2}{|c|}{$\begin{array}{l}\text { OAG } \\
(n 290)\end{array}$} & \multirow[b]{2}{*}{$P+$} \\
\hline & $\%$ or Mean & SD & $\%$ or Mean & SD & $\%$ or Mean & SD & \\
\hline \multicolumn{8}{|l|}{ Potential confounding variables } \\
\hline Sex (male) & $42 \cdot 8$ & - & $50 \cdot 2^{*}$ & - & $46 \cdot 9$ & - & 0.01 \\
\hline Age (years) & $60 \cdot 4$ & $10 \cdot 1$ & $57 \cdot 3^{\star \star}$ & $8 \cdot 9$ & $63 \cdot 3^{\star *}$ & $10 \cdot 7$ & $<0.001$ \\
\hline Current smoking status & $16 \cdot 6$ & - & $16 \cdot 7$ & - & $19 \cdot 0$ & - & 0.59 \\
\hline Sun exposure $(\geq 2 \mathrm{~h} / \mathrm{d})$ & $43 \cdot 7$ & - & $40 \cdot 0$ & - & $43 \cdot 1$ & - & 0.35 \\
\hline Family history of glaucoma & 1.9 & - & $3 \cdot 9^{\star}$ & - & $1 \cdot 7$ & - & 0.02 \\
\hline Migraine & $24 \cdot 3$ & - & $19 \cdot 3^{*}$ & - & $23 \cdot 4$ & - & 0.07 \\
\hline Cold hands & $23 \cdot 4$ & - & $20 \cdot 2$ & - & $27 \cdot 2$ & - & $0 \cdot 10$ \\
\hline $\mathrm{SBP}(\mathrm{mmHg})$ & 125 & 17 & 126 & 18 & $129^{\star \star}$ & 18 & $<0.01$ \\
\hline \multicolumn{8}{|l|}{ Laboratory findings } \\
\hline Fasting glucose (mg/dl) & 101 & 22 & 100 & 23 & 101 & 28 & 0.56 \\
\hline Total cholesterol (mg/dl) & 195 & 37 & 193 & 36 & 192 & 36 & $0 \cdot 13$ \\
\hline TAG (mg/dl) & 142 & 106 & 143 & 91 & 141 & 88 & 0.96 \\
\hline 25(OH)D (ng/ml) & $18 \cdot 7$ & $6 \cdot 6$ & $18 \cdot 4$ & $6 \cdot 0$ & $18 \cdot 1$ & $6 \cdot 5$ & $0 \cdot 17$ \\
\hline Visual acuity (LogMAR) & $0 \cdot 12$ & $0 \cdot 18$ & $0 \cdot 11^{*}$ & $0 \cdot 15$ & $0 \cdot 20^{\star \star}$ & $0 \cdot 26$ & $<0.001$ \\
\hline \multicolumn{8}{|l|}{ Predictive factors for OAG } \\
\hline IOP (mmHg) & $13 \cdot 9$ & $2 \cdot 7$ & $14 \cdot 3^{*}$ & $2 \cdot 8$ & $14 \cdot 7^{\star \star}$ & $3 \cdot 2$ & $<0.01$ \\
\hline Vertical cup-to-disc ratio & 0.35 & $0 \cdot 13$ & $0.57^{\star \star}$ & $0 \cdot 13$ & $0.59^{\star \star}$ & $0 \cdot 19$ & $<0.001$ \\
\hline Horizontal cup-to-disc ratio & 0.35 & $0 \cdot 12$ & $0 \cdot 56^{* *}$ & $0 \cdot 13$ & $0 \cdot 58^{\star *}$ & $0 \cdot 18$ & $<0.001$ \\
\hline
\end{tabular}

OAG, open-angle glaucoma; SBP, systolic blood pressure; 25(OH)D, 25-hydroxyvitamin D; IOP, intraocular pressure.

${ }^{\star} P<0.05,{ }^{\star *} P<0.001 \mathrm{v}$. healthy group.

tThe $\chi^{2}$ test or one-way ANOVA was used. 


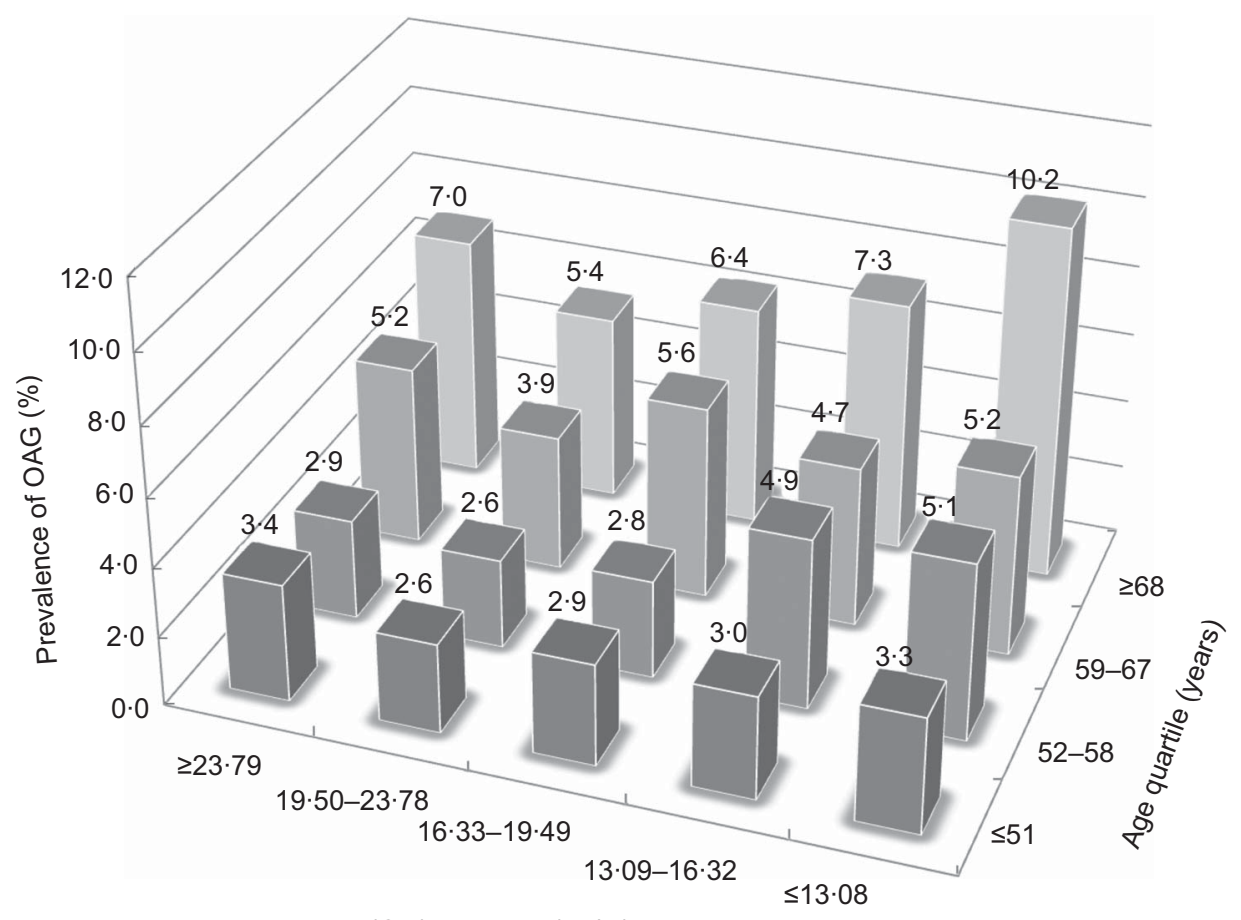

25(OH)D quintile (ng/dl)

Fig. 2 Prevalence of open-angle glaucoma (OAG) calculated per quintile of serum 25-hydroxyvitamin $D(25(O H) D)$ divided by quartile of age among 6094 adult participants in the Fifth Korean National Health and Nutrition Examination Survey (KNHANES V), 2010-2011

OAG and glaucoma suspect or OAG in adjusted analyses by serum $25(\mathrm{OH}) \mathrm{D}$ quintiles. The logistic regression in Model 1 showed that the association between 25(OH)D and OAG was reverse $\mathrm{J}$-shaped ( $P$ for linear trend $=0.05$ and $P$ for quadratic trend $<0 \cdot 01)$. Compared with the second $25(\mathrm{OH}) \mathrm{D}$ quintile (the lowest quintile of $\mathrm{OAG}$ prevalence; Fig. 2), the adjusted odds ratios were 1.26, 1.31, 1.36 and 1.69 in the first, third, fourth and fifth quintiles, respectively. We observed that the fifth quintile (the lowest quintile of serum $25(\mathrm{OH}) \mathrm{D}$ level) had a significantly greater odds ratio referent to the second quintile $(P<0 \cdot 01)$. The relationship between OAG and serum 25(OH)D level was substantially unchanged, even after adjustment for other confounders that were related to vitamin $\mathrm{D}$ status and risk of $\mathrm{OAG}$ (Model 2 and 3). After controlling for all confounding variables, odds ratios of OAG across decreasing quintiles of serum 25(OH)D level also showed a significant quadratic trend with a reverse J-shaped relationship (Model 3, $P$ for quadratic trend $=0 \cdot 02$ ). Since the association was significant after adjustment for IOP (Model 3), these results might indicate an IOP-independent effect of serum 25(OH)D level. After stratifying the data according to sex, significance of the odds ratio trend was observed only in the male group (Model 3, $P$ for quadratic trend $=0 \cdot 01$ ). There was no obvious trend in the female group (Model $3, P$ for quadratic trend $=0 \cdot 39$ ).

When the logistic regression analyses using continuous serum 25(OH)D level were conducted (Table 2), there was a significant change in adjusted odds ratio of OAG for a $1 \mathrm{ng} / \mathrm{dl}$ increase in 25(OH)D (Model 1 , OR $=0.97$, $P=0 \cdot 02)$. This result indicated that the risk of OAG was associated with overall decreases in serum 25(OH)D level, although J-shaped patterns were observed in the above analyses using quintiles of $25(\mathrm{OH}) \mathrm{D}$. To explore the overall association between serum 25(OH)D level and OAG, we used generalized additive models with cubic spline regression (Fig. 3). The smoothed curve generated from the cubic spline model, which was adjusted for variables in the fully adjusted model (Model 3), showed a decrease in odds ratio of OAG as serum $25(\mathrm{OH}) \mathrm{D}$ levels rose in the overall pattern. However, when we excluded the sparse areas with $25(\mathrm{OH}) \mathrm{D}$ values below the 5 th $(\leq 9.97 \mathrm{ng} / \mathrm{dl})$ and above the 95 th $(\geq 30.73 \mathrm{ng} / \mathrm{dl})$ percentiles, we found a reverse J-shaped pattern that was consistent with the quintile analyses. Consistently, there was an obvious J-shaped trend in the male group, but not in the female group.

However, the outcome of glaucoma suspect or OAG showed a weaker association with serum 25(OH)D level than OAG (Table 2). The odds ratios of glaucoma suspect or OAG had a marginally significant linear trend across quintiles of 25(OH)D (Model 1, $P$ for linear trend $=0.06$ and $P$ for quadratic trend $=0 \cdot 12)$. The analyses using continuous serum 25(OH)D level also showed a weak linear association with marginal significance (Model 1, $P=0 \cdot 04)$. 
Table 2 Odds ratios of open-angle glaucoma and glaucoma suspect according to quintiles of serum 25-hydroxyvitamin D among 6094 adult participants in the Fifth Korean National Health and Nutrition Examination Survey (KNHANES V), 2010-2011

\begin{tabular}{|c|c|c|c|c|c|c|c|c|c|c|c|c|c|}
\hline & \multicolumn{11}{|c|}{ Serum 25(OH)D level quintile } & & \\
\hline & \multicolumn{2}{|c|}{$\begin{array}{c}\mathrm{Q1} \\
(\geq 23.79 \mathrm{ng} / \mathrm{dl})\end{array}$} & \multirow{2}{*}{$\begin{array}{c}\text { Q2 } \\
\frac{(19 \cdot 50-23 \cdot 78 \mathrm{ng} / \mathrm{dl})}{\text { OR }}\end{array}$} & \multicolumn{2}{|c|}{$\begin{array}{c}\text { Q3 } \\
(16 \cdot 33-19 \cdot 49 \mathrm{ng} / \mathrm{dl})\end{array}$} & \multicolumn{2}{|c|}{$\begin{array}{c}\mathrm{Q} 4 \\
(13 \cdot 09-16 \cdot 32 \mathrm{ng} / \mathrm{dl})\end{array}$} & \multicolumn{2}{|c|}{$\begin{array}{c}\text { Q5 } \\
(\leq 13 \cdot 08 \mathrm{ng} / \mathrm{dl})\end{array}$} & \multirow{2}{*}{$\begin{array}{l}P \text { for linear } \\
\text { trend }\end{array}$} & \multirow{2}{*}{$\begin{array}{l}P \text { for quadratic } \\
\text { trend }\end{array}$} & \multicolumn{2}{|c|}{$\begin{array}{l}\text { Change in OR for } 1 \mathrm{ng} / \mathrm{d} \text { l } \\
\text { increase in } 25(\mathrm{OH}) \mathrm{D}\end{array}$} \\
\hline & OR & $95 \% \mathrm{Cl}$ & & OR & $95 \% \mathrm{Cl}$ & OR & $95 \% \mathrm{Cl}$ & OR & $95 \% \mathrm{Cl}$ & & & OR & $P$ \\
\hline \multicolumn{14}{|l|}{ Total } \\
\hline \multicolumn{14}{|l|}{ OAG } \\
\hline Model 1† & $1 \cdot 26$ & $0 \cdot 85,1 \cdot 88$ & 1.00 (Ref.) & $1 \cdot 31$ & $0 \cdot 88,1.97$ & $1 \cdot 36$ & $0.91,2 \cdot 03$ & $1 \cdot 69$ & $1 \cdot 14,2 \cdot 49$ & 0.05 & $<0.01$ & 0.97 & 0.02 \\
\hline Model $2 \ddagger$ & $1 \cdot 26$ & $0.85,1 \cdot 88$ & 1.00 (Ref.) & $1 \cdot 30$ & $0.87,1.95$ & $1 \cdot 35$ & $0.90,2.02$ & $1 \cdot 67$ & $1 \cdot 13,2 \cdot 47$ & 0.05 & 0.01 & 0.98 & 0.03 \\
\hline Model $3 \S$ & $1 \cdot 28$ & $0.86,1.90$ & 1.00 (Ref.) & $1 \cdot 32$ & $0.88,1.98$ & $1 \cdot 34$ & $0 \cdot 89,2 \cdot 00$ & $1 \cdot 61$ & $1 \cdot 09,2 \cdot 38$ & 0.08 & 0.02 & 0.98 & 0.04 \\
\hline \multicolumn{14}{|c|}{ Glaucoma suspect or OAG } \\
\hline Model $1+$ & 0.94 & $0.73,1 \cdot 21$ & 1.00 (Ref.) & 0.95 & $0 \cdot 74,1 \cdot 23$ & $1 \cdot 19$ & $0.93,1.52$ & $1 \cdot 14$ & $0 \cdot 89,1 \cdot 47$ & 0.06 & $0 \cdot 12$ & 0.98 & 0.04 \\
\hline Model $2 \ddagger$ & 0.93 & $0.72,1 \cdot 21$ & $1 \cdot 00$ (Ref.) & 0.94 & $0.73,1.22$ & $1 \cdot 19$ & $0.93,1.52$ & $1 \cdot 13$ & $0.88,1.46$ & 0.06 & $0 \cdot 13$ & 0.98 & 0.04 \\
\hline \multirow{2}{*}{\multicolumn{14}{|c|}{ Males }} \\
\hline & & & & & & & & & & & & & \\
\hline \multicolumn{14}{|l|}{ OAG } \\
\hline Model 1† & $1 \cdot 51$ & $0 \cdot 87,2 \cdot 63$ & 1.00 (Ref.) & $1 \cdot 46$ & $0 \cdot 81,2 \cdot 64$ & $1 \cdot 86$ & $1 \cdot 04,3 \cdot 35$ & $2 \cdot 23$ & $1 \cdot 24,4 \cdot 03$ & 0.04 & $<0.01$ & 0.97 & 0.09 \\
\hline Model $2 \ddagger$ & $1 \cdot 50$ & $0 \cdot 86,2 \cdot 62$ & 1.00 (Ref.) & $1 \cdot 47$ & $0 \cdot 82,2 \cdot 66$ & $1 \cdot 88$ & $1 \cdot 05,3 \cdot 38$ & $2 \cdot 25$ & $1 \cdot 25,4 \cdot 07$ & 0.03 & $<0.01$ & 0.97 & 0.09 \\
\hline Model $3 \S$ & $1 \cdot 49$ & $0 \cdot 85,2 \cdot 61$ & 1.00 (Ref.) & $1 \cdot 50$ & $0 \cdot 83,2 \cdot 71$ & $1 \cdot 87$ & $1 \cdot 04,3 \cdot 38$ & $2 \cdot 16$ & $1 \cdot 19,3.92$ & 0.05 & 0.01 & 0.97 & $0 \cdot 11$ \\
\hline \multicolumn{14}{|c|}{ Glaucoma suspect or OAG } \\
\hline Model 1† & 0.99 & $0 \cdot 70,1 \cdot 38$ & 1.00 (Ref.) & 1.05 & $0 \cdot 74,1 \cdot 49$ & $1 \cdot 30$ & $0.92,1.85$ & $1 \cdot 12$ & $0 \cdot 76,1 \cdot 64$ & 0.23 & 0.34 & 0.98 & $0 \cdot 14$ \\
\hline Model $2 \ddagger$ & 0.97 & $0.69,1.36$ & 1.00 (Ref.) & 1.05 & $0.74,1.49$ & $1 \cdot 30$ & $0.91,1.85$ & $1 \cdot 11$ & $0.75,1.63$ & 0.22 & 0.36 & 0.98 & $0 \cdot 14$ \\
\hline Model $3 \S$ & 0.98 & $0.70,1.37$ & 1.00 (Ref.) & 1.05 & $0 \cdot 74,1 \cdot 49$ & $1 \cdot 30$ & $0.91,1.85$ & $1 \cdot 10$ & $0.74,1.62$ & $0 \cdot 25$ & 0.38 & 0.98 & $0 \cdot 16$ \\
\hline \multicolumn{14}{|l|}{ Females } \\
\hline \multicolumn{14}{|l|}{ OAG } \\
\hline Model 1t & 1.09 & $0.61,1.93$ & $1 \cdot 00$ (Ref.) & $1 \cdot 17$ & $0.67,2.03$ & 1.02 & $0.59,1.77$ & $1 \cdot 35$ & $0.81,2 \cdot 26$ & 0.38 & 0.28 & 0.98 & $0 \cdot 16$ \\
\hline Model 2‡ & 1.09 & $0.61,1.93$ & 1.00 (Ref.) & $1 \cdot 17$ & $0.67,2.03$ & $1 \cdot 02$ & $0.59,1.77$ & $1 \cdot 32$ & $0 \cdot 79,2 \cdot 22$ & 0.41 & $0 \cdot 30$ & 0.98 & $0 \cdot 17$ \\
\hline \multirow{2}{*}{\multicolumn{14}{|c|}{ Glaucoma suspect or OAG }} \\
\hline & & & & & & & & & & & & & \\
\hline Model $1+$ & 0.92 & $0.62,1.37$ & 1.00 (Ref.) & 0.86 & $0.59,1.26$ & 1.09 & $0.77,1.55$ & $1 \cdot 16$ & $0 \cdot 82,1.62$ & $0 \cdot 18$ & 0.24 & 0.98 & $0 \cdot 18$ \\
\hline Model 2‡ & 0.93 & $0.63,1.37$ & 1.00 (Ref.) & $0 \cdot 86$ & $0.59,1 \cdot 26$ & 1.09 & $0.77,1.55$ & $1 \cdot 15$ & $0.81,1.62$ & $0 \cdot 18$ & 0.26 & 0.98 & $0 \cdot 19$ \\
\hline Model $3 \S$ & 0.95 & $0.64,1.41$ & 1.00 (Ref.) & 0.87 & $0 \cdot 60,1 \cdot 26$ & 1.08 & $0.76,1.53$ & $1 \cdot 11$ & $0.79,1.57$ & $0 \cdot 19$ & 0.34 & 0.99 & $0 \cdot 31$ \\
\hline
\end{tabular}

25(OH)D, 25-hydroxyvitamin D; OAG, open-angle glaucoma; Ref., referent category.

+Model 1 was adjusted for sex, age, current smoking status, sun exposure, family history of glaucoma, migraine and cold hands.

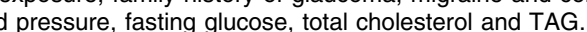

$\S$ Model 3 was adjusted for variables in Model 2 plus visual acuity and intraocular pressure. 

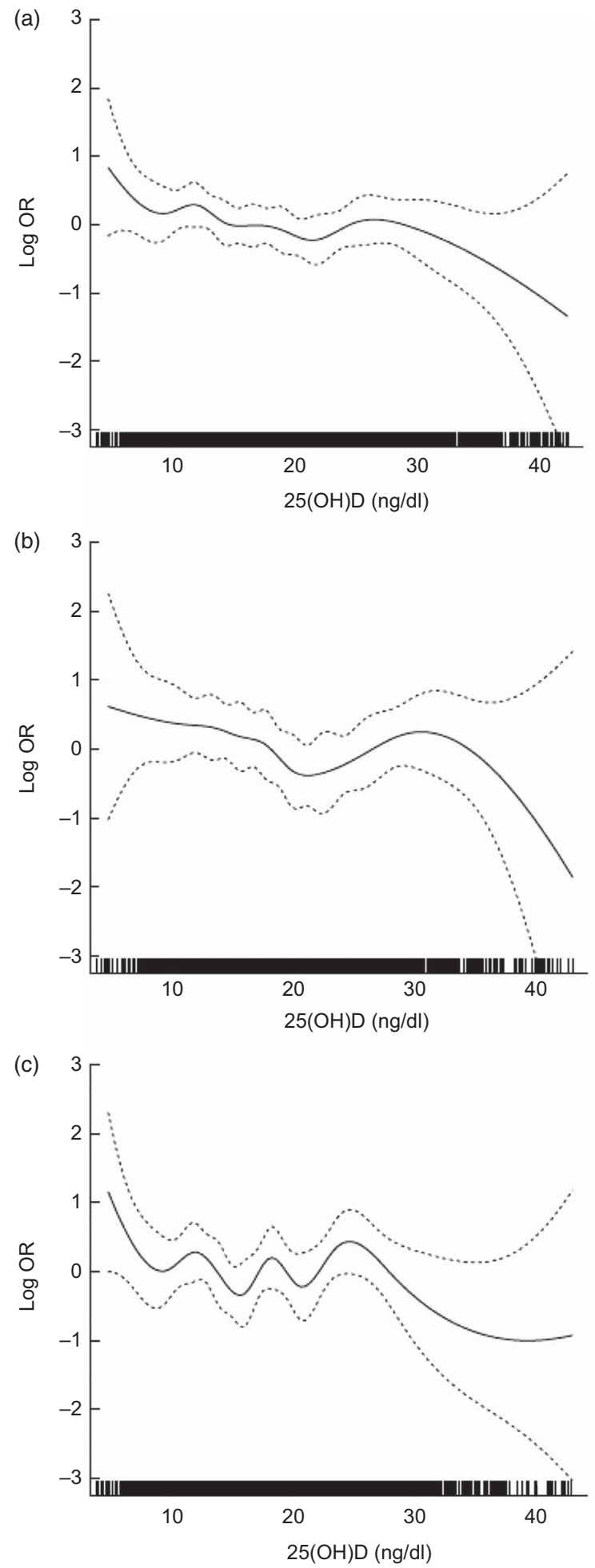

Fig. 3 Adjusted log odds ratios (-) with $95 \%$ confidence intervals (- - - -) for risk of open-angle glaucoma by serum 25hydroxyvitamin D $(25(\mathrm{OH}) \mathrm{D})$, using generalized additive models with cubic spline regression, among (a) the total, (b) the male and (c) the female participants, Fifth Korean National Health and Nutrition Examination Survey (KNHANES V), 2010-2011. The model was adjusted for sex, age, current smoking status, sun exposure, family history of glaucoma, migraine, cold hands, systolic blood pressure, fasting glucose, total cholesterol, TAG, visual acuity and intraocular pressure
In addition, we examined the interaction between serum $25(\mathrm{OH}) \mathrm{D}$ and predictive factors of OAG. The adjusted geometric means of IOP and vertical and horizontal cup-todisc ratios of the optic nerve head are shown in Table 3. The trends of the predictive factors were linear rather than quadratic across the quintiles of $25(\mathrm{OH}) \mathrm{D}$. The multivariable linear regression models measured the associations of serum 25(OH)D level with these factors. Serum 25(OH)D levels were inversely and consistently related with vertical $(P=0 \cdot 03)$ and horizontal $(P=0 \cdot 01)$ cup-to-disc ratios even after full adjustment. This finding suggests that the structural change of the optic nerve, an important factor for detection of glaucoma, may be affected by $25(\mathrm{OH}) \mathrm{D}$ levels. We also observed a significant inverse relationship between IOP and serum 25(OH)D levels $(P<0 \cdot 001)$.

\section{Discussion}

To our knowledge, the present study is the first to demonstrate an association between vitamin D status and risk of OAG in a human population sample. The nature of this relationship was a J-shaped increase of OAG at lower vitamin $D$ status. In the study population, taken from KNHANES V, the cross-sectional findings indicate that the lowest quintile of serum 25(OH)D level had a significantly increased odds ratio of OAG, but not glaucoma suspect or OAG, suggesting that vitamin D deficiency may be significantly linked to clinical optic nerve dysfunction of OAG patients, rather than simply structural glaucomatous change of glaucoma suspects. Unexpectedly, there was an insignificantly increased risk of OAG in the highest quintile of serum $25(\mathrm{OH})$ level, and it contributed to a statistically significant quadratic association. In addition, we found that IOP, vertical and horizontal cup-to-disc ratios had a significant relationship to serum 25(OH)D level. These results support the study's hypothesis that low vitamin D status is associated with an increased risk of OAG. Since the analyses were adjusted for a wide range of confounders including visual acuity, sun exposure and metabolic factors, vitamin D deficiency should be considered as an independent risk factor for OAG. Low vitamin D status might render additional hazardous effect on the optic nerve because patients with OAG already have impaired vision that might reduce daily activities and deteriorate vitamin D status. Therefore, it is possible to describe that patients with OAG might be in a vicious cycle of visual impairment and low vitamin D status (Fig. 4).

The association between vitamin $\mathrm{D}$ status and OAG is not fully understood. Previous studies have mostly focused on the relationship between vitamin $\mathrm{D}$ and $\mathrm{IOP}^{(26)}$. Although some animal studies suggest that vitamin D may be associated with IOP, the results of human studies have been equivocal ${ }^{(27)}$. In a randomized controlled trial, there was no reduction in IOP in human subjects with high-dose vitamin D supplement ${ }^{(26)}$. However, this result 
might be limited by the small sample size consisting of only eighty-seven healthy participants and a 6-month short-term follow-up. Our findings showed that IOP was associated with serum $25(\mathrm{OH}) \mathrm{D}$ level in a populationbased approach including participants with OAG and glaucoma suspect, as well as healthy controls. Therefore, further work is required to confirm whether vitamin D status affects the development of OAG through IOP. In addition, a previous epidemiological study reported that the risk of OAG due to exfoliation syndrome increased in northern-latitude populations, suggesting reduced vitamin D metabolism could be one of mechanisms explaining the link between latitude and exfoliation syndrome ${ }^{(28)}$. However, these data are insufficient to support our findings because the prevalence of exfoliation syndrome is very low in Asian countries including South $\mathrm{Korea}^{(29)}$.

Recently, OAG is seen as a complex and multifactorial disease that may be influenced by not only IOP, but also the systemic conditions. Since effects of low serum $25(\mathrm{OH}) \mathrm{D}$ level on the risk of OAG seem to be significant in the adjusted models, vitamin D may have an IOP-independent protective effect on OAG. The potential mechanisms underlying this association are still not fully understood, but may be mediated by the several protective roles of vitamin D, either directly (by activation of the vitamin $\mathrm{D}$ receptor) or indirectly (by regulation of calcium homeostasis ${ }^{(30,31)}$. First, vitamin D may affect immunomodulation in the pathogenesis of $\mathrm{OAG}^{(3)}$. Recent researches have shown that imbalance of the immune system is a major contributor to neurodegenerative injuries on the optic nerve axons and ganglion cell bodies. Since it has been well established that vitamin D has marked effects on regulating immune cell functions, this effect may play a key role in the protection of the optic nerve. Second, vitamin D also regulates important neurotrophic factors in the central nervous system and the plasticity process in neural networks. The researches using animal models have found that vitamin D has the neurotrophic property associated with the synthesis of neurotrophic growth factors and neurotransmitter metabolism $^{(9,10)}$. It is possible that this effect may help in the regeneration of the optic nerve after injury. Third, vitamin $\mathrm{D}$ regulates oxidative stress on neurons by activation of calcium channels ${ }^{(31)}$. A previous study suggests that the oxidative stress-induced amyloid $\beta$-peptide deposition could involve the optic nerve in $\mathrm{OAG}^{(6)}$. In support of this view, evidence accumulating over two decades has shown that vitamin D is closely associated with a number of neurodegenerative or psychiatric diseases such as Alzheimer's disease, Parkinson's disease, depression and schizophrenia ${ }^{(31)}$. Several researches have shown that these diseases could affect the development of OAG; therefore, we have expected that OAG shares the biological mechanisms of vitamin $\mathrm{D}$.

Another possible explanation for the association of lower vitamin D status with OAG may lie in the mechanism of 


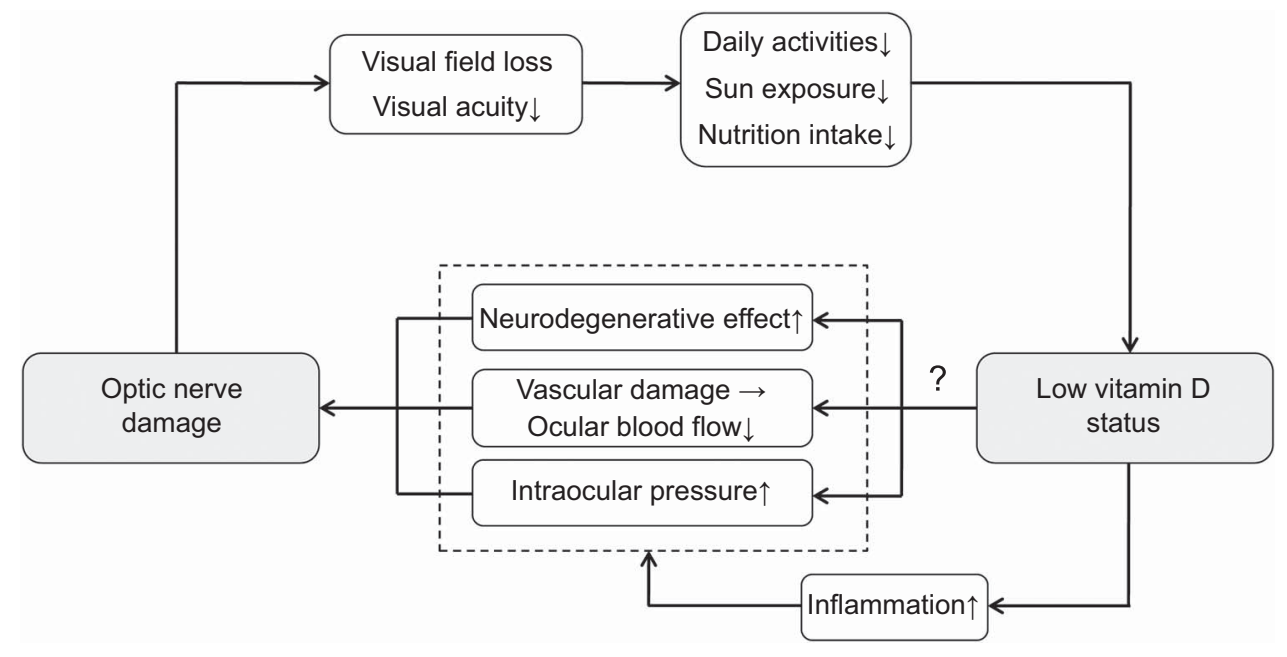

Fig. 4 Diagram for the possible relationship between optic nerve damage and reduced vitamin D status

impaired ocular blood flow. Several studies have shown the influence of vitamin D on peripheral or micro-vessel circulation $^{(30)}$. These studies have revealed that vitamin D regulates the rennin-angiotensin system and improves endothelial cell-dependent vessel vasodilatation. In one animal-based study, it is discussed that suppression of the rennin-angiotensin system can decrease the risk of OAG by improving ocular blood flow ${ }^{(32)}$. An anti-inflammatory effect of vitamin D also inhibits endothelial dysfunction from metabolic damage or oxidative stress ${ }^{(30)}$. Furthermore, vitamin D status might reflect chronic non-specific illness that could affect systemic circulation ${ }^{(33)}$. These researches have shown that improving vitamin D status may have a beneficial effect on the vascular dysregulation leading to local vasospasm ${ }^{(5,33)}$.

Despite expectations that a linear trend would appear, we actually found a reverse J-shaped relationship between serum $25(\mathrm{OH}) \mathrm{D}$ level and OAG. It is feasible that the relationship identified in the present study could reflect exposure to UV light, because there is a theoretical basis for assuming that long-term exposure to UV not only increases vitamin D synthesis but also directly induces oxidative damage to the anterior chamber of the eye, finally leading to $O A G^{(34)}$. Therefore, it is possible that highly elevated vitamin D levels could be associated with OAG due to excessive exposure to UV. Another possible explanation is that there may be a subgroup of the population who is genetically resistant to vitamin $\mathrm{D}^{(35)}$. These individuals could have relatively elevated levels of serum $25(\mathrm{OH}) \mathrm{D}$, but may be accompanied by relative vitamin $\mathrm{D}$ deficiency.

We also observed the unexpected finding that there was no obvious trend between vitamin $\mathrm{D}$ and $\mathrm{OAG}$ in females. The disturbed trend among females is most likely explained by menstrual status or hormone replacement therapy. In general, females have an independent risk factor for OAG due to lower levels of oestrogen and progesterone after menopause, while males may not ${ }^{(36)}$.
Furthermore, recent works have revealed that sexual hormonal status could lead to nutrition deficiency and various chronic diseases, which may be linked to low vitamin D status and development of $\mathrm{OAG}^{(37,38)}$.

The strength of present study is that it is based on a large population sample size so it minimizes selection bias. An additional strength is the characterization of demographic, biochemical and ophthalmologic covariates to reveal an independent association between serum 25(OH)D level and OAG. The study has several limitations. First, the findings reported herein are cross-sectional and observational. Although OAG is usually considered irreversible and the study population is nationwide and randomly sampled, the study's cross-sectional nature precludes the determination of a direct association between vitamin D and OAG. We expect that a longitudinal and interventional study design will draw a definitive conclusion about the association between vitamin D status and OAG. Second, the ISGEO criteria for OAG diagnosis did not consider the progression of disease and comprehensive ophthalmologic examination. Although use of the ISGEO criteria is a preferred method in a cross-sectional epidemiological study, clinical diagnosis of OAG may not perfectly match the ISGEO criteria $^{(19,20)}$. Third, this is an Asia-specific study performed at the level of a single country. Generally, the incidence and progression of glaucoma are influenced by ethnic differences and genetic backgrounds. Thus, it is uncertain whether the results will be equally applicable to other demographic groups.

\section{Conclusion}

There was a reverse J-shaped association between vitamin D status and the risk of OAG, with significantly elevated risk at lower vitamin D levels. This epidemiological finding, which is not previously reported, suggests that 
vitamin D deficiency should be considered as a potential risk factor for the development of OAG. In order to confirm the protective effect on OAG, larger, more comprehensive studies of vitamin D supplementation are needed.

\section{Acknowledgements}

Sources of funding: The authors have no support or funding to report. Conflicts of interest: The authors declare that they have no conflict of interest. Ethical approval: The study protocol was approved by the institutional review board at the Korea Centers for Disease Control and Prevention (IRB No: 2010-02CON-21-C, 2011-02CON-06-C). Authors' contributions: T.K.Y. performed the statistical analysis and drafted the manuscript. E.O. collected the data and analysed the experimental results. S.H. collected the data and provided feedback on the paper. T.K.Y. and E.O. contributed equally to this work.

\section{References}

1. Quigley HA \& Broman AT (2006) The number of people with glaucoma worldwide in 2010 and 2020. Br J Ophthalmol 90, 262-267.

2. Quigley HA (2011) Glaucoma. Lancet 377, 1367-1377.

3. McKinnon SJ (2012) The cell and molecular biology of glaucoma: common neurodegenerative pathways and relevance to glaucoma. Invest Ophthalmol Vis Sci 53, 2485-2487.

4. Yanagi M, Kawasaki R, Wang JJ et al. (2011) Vascular risk factors in glaucoma: a review. Clin Experiment Ophthalmol 39, 252-258.

5. Flammer J \& Orgül S (1998) Optic nerve blood-flow abnormalities in glaucoma. Prog Retin Eye Res 17, 267-289.

6. McKinnon SJ (2003) Glaucoma: ocular Alzheimer's disease? Front Biosci 8, s1140-s1156.

7. Hossein-Nezhad A \& Holick MF (2013) Vitamin D for health: a global perspective. Mayo Clin Proc 88, 720-755.

8. Tarcin O, Yavuz DG, Ozben B et al. (2009) Effect of vitamin $D$ deficiency and replacement on endothelial function in asymptomatic subjects. I Clin Endocrinol Metab 94, 4023-4030.

9. Balion C, Griffith LE, Strifler L et al. (2012) Vitamin D, cognition, and dementia: a systematic review and metaanalysis. Neurology 79, 1397-1405.

10. Van der Schaft J, Koek HL, Dijkstra E et al. (2013) The association between vitamin $\mathrm{D}$ and cognition: a systematic review. Ageing Res Rev 12, 1013-1023.

11. Schreiner DS, Jande SS \& Lawson DE (1985) Target cells of vitamin D in the vertebrate retina. Acta Anat (Basel) 121, 153-162.

12. Lucas RM, Ponsonby A-L, Dear K et al. (2011) Sun exposure and vitamin $\mathrm{D}$ are independent risk factors for CNS demyelination. Neurology 76, 540-548.

13. Oh SW, Lee S, Park C et al. (2005) Elevated intraocular pressure is associated with insulin resistance and metabolic syndrome. Diabetes Metab Res Rev 21, 434-440.

14. Read RM \& Spaeth GL (1974) The practical clinical appraisal of the optic disc in glaucoma: the natural history of cup progression and some specific disc-field correlations. Trans Am Acad Ophthalmol Otolaryngol $\mathbf{7 8}$, OP255-OP274.

15. Oh K, Lee J, Lee B et al. (2007) Plan and operation of the 4th Korea National Health and Nutrition Examination Survey (KNHANES IV). Korean J Epidemiol 29, 139-145.
16. Ekström C (2008) Incidence of open-angle glaucoma in central Sweden. Acta Ophthalmol 86, 747-754.

17. Ramrattan RS, Wolfs RC, Panda-Jonas S et al. (2001) Prevalence and causes of visual field loss in the elderly and associations with impairment in daily functioning: the Rotterdam Study. Arch Ophthalmol 119, 1788-1794.

18. Yoon K-C, Mun G-H, Kim S-D et al. (2011) Prevalence of eye diseases in South Korea: data from the Korea National Health and Nutrition Examination Survey 2008-2009. Korean J Opbthalmol 25, 421-433.

19. Foster PJ, Buhrmann R, Quigley HA et al. (2002) The definition and classification of glaucoma in prevalence surveys. Br J Ophthalmol 86, 238-242.

20. Chon B, Qiu M \& Lin SC (2013) Myopia and glaucoma in the South Korean population. Invest Ophthalmol Vis Sci $\mathbf{5 4}$, 6570-6577.

21. Choi C-J, Seo M, Choi W-S et al. (2013) Relationship between serum 25-hydroxyvitamin $\mathrm{D}$ and lung function among Korean adults in Korea National Health and Nutrition Examination Survey (KNHANES), 2008-2010. J Clin Endocrinol Metab 98, 1703-1710.

22. Kim M, Kim T-W, Park KH et al. (2012) Risk factors for primary open-angle glaucoma in South Korea: the Namil study. Jpn J Ophthalmol 56, 324-329.

23. Hart PM, Chakravarthy U, MacKenzie G et al. (1996) Teletherapy for subfoveal choroidal neovascularisation of age-related macular degeneration: results of follow up in a non-randomised study. $\mathrm{Br} J$ Ophthalmol 80, 1046-1050.

24. Rubin GS, Roche KB, Prasada-Rao P et al. (1994) Visual impairment and disability in older adults. Optom Vis Sci $\mathbf{7 1}$, 750-760.

25. Mukamal KJ, Chung H, Jenny NS et al. (2005) Alcohol use and risk of ischemic stroke among older adults the cardiovascular health study. Stroke 36, 1830-1834.

26. Krefting EA, Jorde R, Christoffersen T et al. (2013) Vitamin $\mathrm{D}$ and intraocular pressure - results from a case-control and an intervention study. Acta Ophthalmol (Epublication ahead of print version).

27. Kutuzova GD, Gabelt BT, Kiland JA et al. (2012) $1 \alpha$, 25-Dihydroxyvitamin $\mathrm{D}(3)$ and its analog, 2-methylene19-nor-(20S)-1 $\alpha, 25$-dihydroxyvitamin D(3) (2MD), suppress intraocular pressure in non-human primates. Arch Biochem Biophys 518, 53-60.

28. Wiggs JL (2012) The cell and molecular biology of complex forms of glaucoma: updates on genetic, environmental, and epigenetic risk factors. Invest Ophthalmol Vis Sci $\mathbf{5 3}$, 2467-2469.

29. Chen H, Chen LJ, Zhang M et al. (2010) Ethnicity-based subgroup meta-analysis of the association of LOXL1 polymorphisms with glaucoma. Mol Vis 16, 167-177.

30. Yang L, Ma J, Zhang X et al. (2012) Protective role of the vitamin D receptor. Cell Immunol 279, 160-166.

31. DeLuca GC, Kimball SM, Kolasinski J et al. (2013) Review: the role of vitamin $\mathrm{D}$ in nervous system health and disease. Neuropathol Appl Neurobiol 39, 458-484.

32. Shah GB, Sharma S, Mehta AA et al. (2000) Oculohypotensive effect of angiotensin-converting enzyme inhibitors in acute and chronic models of glaucoma. J Cardiovasc Pharmacol 36, 169-175.

33. Pittas AG, Chung M, Trikalinos $T$ et al. (2010) Systematic review: vitamin $\mathrm{D}$ and cardiometabolic outcomes. Ann Intern Med 152, 307-314.

34. Izzotti A, Saccà SC, Longobardi M et al. (2009) Sensitivity of ocular anterior chamber tissues to oxidative damage and its relevance to the pathogenesis of glaucoma. Invest Ophthalmol Vis Sci 50, 5251-5258.

35. McGrath JJ, Eyles DW, Pedersen CB et al. (2010) Neonatal vitamin $\mathrm{d}$ status and risk of schizophrenia: a population- 
based case-control study. Arch Gen Psychiatry 67, 889-894.

36. Pasquale LR, Loomis SJ, Weinreb RN et al. (2013) Estrogen pathway polymorphisms in relation to primary open angle glaucoma: an analysis accounting for gender from the United States. Mol Vis 19, 1471-1481.
37. Lim SK, Kung AWC, Sompongse S et al. (2008) Vitamin D inadequacy in postmenopausal women in Eastern Asia. Curr Med Res Opin 24, 99-106.

38. Battaglia C, Mancini F, Regnani G et al. (2004) Hormone therapy and ophthalmic artery blood flow changes in women with primary open-angle glaucoma. Menopause 11, 69-77. 